\title{
Hydrolytic Co-condensation of Phenyltriethoxysilane with $\gamma$-Aminopropyltriethoxysilane in the Presence of Sodium Dodecyl Sulfate
}

\author{
Shumei LIU, ${ }^{1}$ Hua YE, ${ }^{1,2}$ Yongsheng ZHOU, ${ }^{1}$ and Jianqing ZHAO ${ }^{1,2, \dagger}$ \\ ${ }^{1}$ College of Materials Science and Engineering, South China University of Technology, 510640 Guangzhou, China \\ ${ }^{2}$ The Key Laboratory of GD for High Property and Functional Macromolecular Materials, \\ South China University of Technology, Guangzhou 510640, China
}

(Received August 5, 2005; Accepted October 4, 2005; Published March 15, 2006)

\begin{abstract}
The hydrolytic co-condensation of hydrophobic phenyltriethoxysilane (PTES) and hydrophilic $\gamma$ aminopropyltriethoxysilane (APS) under basic catalysis in the presence of sodium dodecyl sulfate (SDS) was investigated. Copolymer particles were influenced significantly by APS/PTES molar ratios, total monomer and SDS concentration. Amino group content of the copolymer was determined by element analysis and back titration. Scanning electron micrographs revealed the morphologies of the copolymer particles. FT-IR, solid state ${ }^{29} \mathrm{Si}$ NMR and TGA were used to characterize the copolymer. [DOI 10.1295/polymj.38.220] KEY WORDS $\gamma$-Aminopropyltriethoxysilane / Phenyltriethoxysilane / Sodium Dodecyl Sulfate / Hydrolytic Co-condensation / Copolymer Particles /
\end{abstract}

Poly(phenylsilsesquioxane) (PPSQ) from the hydrolytic condensation of phenyltriethoxysilane exhibits outstanding thermal stability, good solubility in common organic solvents and fine electric insulting properties, ${ }^{1,2}$ but its compatibility and reactivity with other polymers are not good enough. Poly(aminopropylsilsesquioxane) (PASQ) from the hydrolytic condensation of APS has been developed rapidly as a precursor to organic/inorganic hybrid polymers, ${ }^{3}$ as starting material of a two-dimensional polysiloxane complex ${ }^{4}$ and as a core for starburst dendrimers due to reactive amino groups, ${ }^{5}$ but it is highly hygroscopic. Many properties of polylsilsesquioxanes can be modified by combining two or more $\mathrm{RSiO}_{3 / 2}$ components ( $\mathrm{R}$ is hydrogen or any alkyl, alkylene, aryl, arylene, or their organo-functional derivatives). ${ }^{6,7}$ The co-condensation method enables better control of the density of functional groups, then control of properties. It is expected that the co-condensation of PTES and APS will yield novel functional silsesquioxanes and the hybrid silsesquioxane will combine the advantages of PPSQ with APSQ. Tunney's patent claimed the preparation of such a poly(aminopropyl/phenylsilsesquioxane) (PAPSQ) by the hydrolytic co-condensation and its use as a precursor of organic/inorganic hybrid materials by imidization or amidation, ${ }^{8}$ but no experimental details or characterization data were supplied. Their scheme under acid catalyst employs expensive tetrahydrofuran (THF) as co-solvent and isolation and purification is complicated. PAPSQ has been investigated in a Stöber-like method in our laboratory, ${ }^{9}$ but some shortcomings such as low amino group contents and large particle diameter are insuperable from the method.

This paper presents a useful alternative for the control of particle size and amino content of the copolymer, and provides easy access to a polysilsesquioxane copolymer with a relatively high degree of functionality. Anionic surfactant SDS was used, which has been shown effective in controlling the size of poly(phenylsilsesquioxane $)^{10}$ and poly(phenyl/methylsilsesquioxane) particles. ${ }^{11}$ Spheroidal copolymer particles with high amino group content are prepared by the hydrolytic co-condensation of hydrophobic PTES and hydrophilic APS in the presence of SDS. Knowledge of these materials, coupled with a wide range of trialkoxyorganosilanes, this simple co-condensation route opens the door for the further development of multifunctional materials.

\section{EXPERIMENTAL}

\section{Materials}

APS ( $\geq 97 \%$, Jiangsu Yizheng Xinde Auxiliary Agent Plant) and PTES (99\%, Dalian Yuanyong Organic Silicon Plant) were used without further purification. SDS and tetramethylammonium hydroxide (TMAOH, 25\%) were of analytical reagent quality.

\section{Preparation of PAPSQ}

A mixture of $20 \mathrm{wt} \%$ APS and PTES (relative to water by weight, the same below) at different molar ratios was added dropwise within $2.5-3 \mathrm{~h}$ to an aqueous solution containing $1 \mathrm{wt} \% \mathrm{SDS}$ and $0.25 \mathrm{wt} \%$

${ }^{\dagger}$ To whom correspondence should be addressed (Tel: +86-20-87113576, Fax: +86-20-87113576, E-mail: psjqzhao@ @scut.edu.cn). 
Table I. Process and results of amino group content (C) of products at various APS mol \% in the feed

\begin{tabular}{cclcc}
\hline & APS & & \multicolumn{2}{c}{ Found $\mathrm{C}(\mathrm{mmol} / \mathrm{g})$} \\
Samples & $(\mathrm{mol} \%)$ & System state & Back titration & Elemental analysis \\
\hline $\mathrm{SA}_{1}$ & 0 & translucent gel & & \\
$\mathrm{SA}_{2}$ & 25 & white latex & & \\
$\mathrm{SA}_{3}$ & 33 & precipitate & 2.12 & 2.17 \\
$\mathrm{SA}_{4}$ & 50 & precipitate & 2.88 & 2.89 \\
$\mathrm{SA}_{5}$ & 75 & flocculent precipitate & 4.05 & 4.12 \\
$\mathrm{SA}_{6}$ & 100 & clear solution & & \\
\hline
\end{tabular}

ATMOH under vigorous agitation. Stirring was stopped at $8 \mathrm{~h}$, and the solution was aged at room temperature overnight. The resultant precipitates were isolated by centrifugation, redispersed in distilled water and centrifuged again to remove SDS. The rinsed product was thoroughly dried under vacuum for $20 \mathrm{~h}$ at $50^{\circ} \mathrm{C}$.

\section{Characterization}

Elemental analysis was performed on a Heraeus CHN-O-RAPID elemental analyzer. FT-IR spectra were recorded on a Vector 33 FT-IR spectrophotometer. The samples were mixed with potassium bromide and pressed to discs. Solid-state samples were performed on a Bruker AVANCE Digital $400 \mathrm{MHz}$ NMR spectrometer. ${ }^{29} \mathrm{Si}$ CP-MAS NMR spectra were measured at $79.49 \mathrm{MHz}$ with a $2.5-\mathrm{s}$ pulse delay, a $2.0-\mathrm{ms}$ contact time and a spinning speed of $6 \mathrm{kHz}$. Chemical shifts of silicon atoms in silsesquioxane compounds were referred to using traditional terminology $\mathrm{T}^{\mathrm{n}}$, where the superscript corresponded to the number of oxygen bridges to other silicon atoms. Thus, an uncondensed monomer was designated $\mathrm{T}^{\circ}$, and a fully condensed polymer with no residual silanols was comprised of $\mathrm{T}^{3}$ silicon atoms. TGA was performed on a Perkin-Elmer TGA-7 thermal analyzer under flowing nitrogen at a heating rate of $20^{\circ} \mathrm{C} / \mathrm{min}$. The morphology of the particles was examined from the product powder dispersed in $1 \mathrm{wt} \%$ SDS aqueous solution by using a Hitachi S-510 scanning electron microscope (SEM).

Quantitative determination of amino groups in the particles was done by back titration. ${ }^{12}$ A typical example was as follows: $0.5 \mathrm{~g}$ powder and $20 \mathrm{~mL}$ of $0.2 \mathrm{M}$ $\mathrm{HCl}$ aqueous solution were charged into a $100-\mathrm{mL}$ flask and the mixture was stirred over $3 \mathrm{~h}$ with a magnetic stirrer at room temperature. After the reaction, the mixture was filtered and the filtrate was titrated with aqueous solution of sodium hydroxide using phenolphtalein as indicator.

\section{RESUITS AND DISCUSSION}

The hydrolytic co-condensation of PTES and APS was conducted at various APS mole percentages maintaining $20 \mathrm{wt} \%$ total monomer concentration. The hydrolytic condensation of PTES produced translucent colloidal solution and tended to gel in high PTES concentration. A white latex was formed when $25 \mathrm{~mol} \%$ APS was contained in the feed. A precipitate yielded with APS in the feed up to $33 \mathrm{~mol} \%$. When APS above $75 \mathrm{~mol} \%$, the precipitate got flocculent. APS alone gave rise to a clear solution. Amino group content of the precipitate products $(\mathrm{C}, \mathrm{mmol} / \mathrm{g})$ determined by element analysis and by back titration increased with APS in the feed and was higher compared with the reported method. ${ }^{9}$ The process and the results are shown in Table I.

Variation with increasing APS may originate from dissolving rate differences between APS and PTES. Immiscible PTES is dispersed into fine droplets as reservoirs of monomer in aqueous phase, depending on the solubilization of SDS. The hydrolysis and condensation of PTES induces a large number of fine particles negatively surface-charged due to the adsorption of anionic SDS. The formed particles separate, not coagulate, and aggregate gels at high PTES concentration. APS with hydrophilic amino groups dissolves in water. Water-soluble oligomer silsesquioxanes are thus formed and a clear solution is obtained. The hydrolysis and condensation rate of PTES increases in the presence of water-soluble APS. The number of primary particles increases correspondingly and aggregation between primary particles accelerates. A certain amount of hydrophilic aminopropyl groups is incorporated into the silsesquioxane networks due to spontaneous intermolecular polycondensation of silanol functional oligomers in the presence of high basicity and large excess of water, both of which favor hydrolysis. Aminopropyl groups seem mainly incorporated onto the surface of the particles due to high hydrophilicity. The copolymer with hydrophilic aminopropyl groups has greater critical chain length for precipitation than the polymer of PTES. As a consequence, larger particles are produced. This is the reason of appearance of white latexes after small amounts of APS added and precipitate with further increase in APS percentages. Finally the incorporation of more aminopropyl groups induces the growing co- 


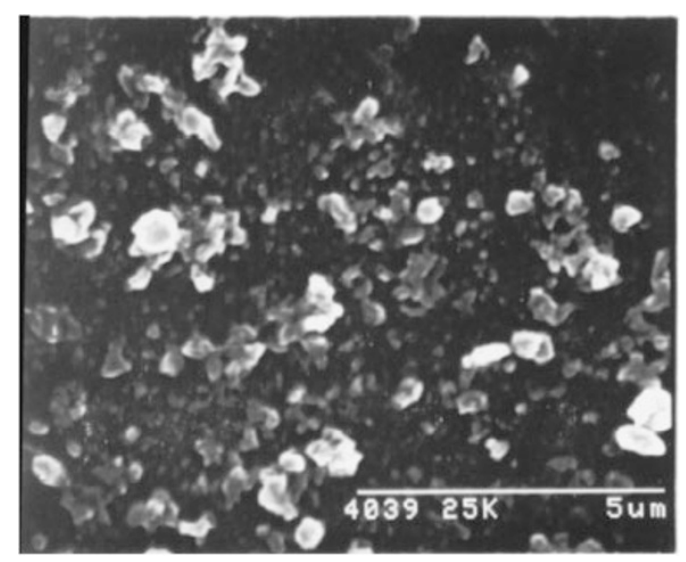

(a)

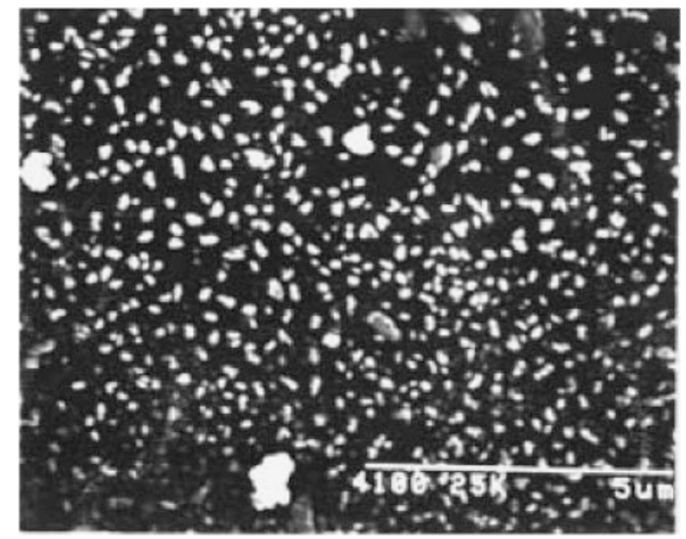

(b)

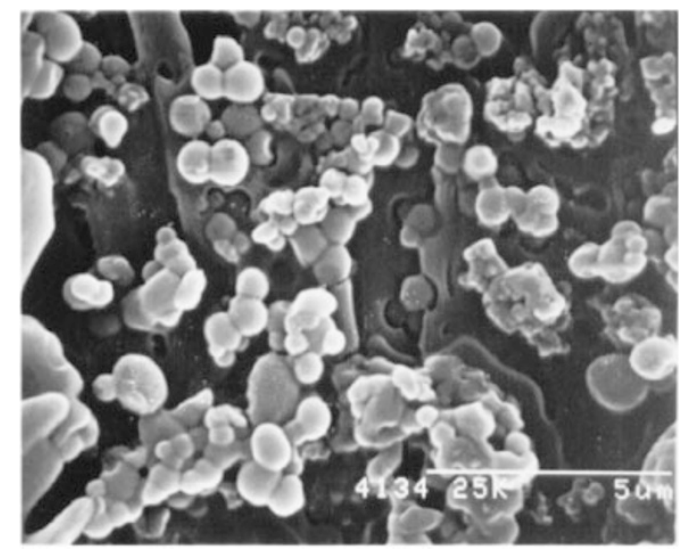

(c)

Figure 1. SEM images of samples (a) $\mathrm{SA}_{3}$; (b) $\mathrm{SA}_{4}$; (c) $\mathrm{SA}_{5}$.

polymer particles to remain very high in hydrophilicity while the hydrophobic phenyl fraction and PTES concentration decrease, resulting in slower nucleation rate and smaller quantity of particles. Therefore flocculent deposition occurs when APS up to $75 \mathrm{~mol} \%$. An appropriate APS/PTES ratio results in the occurrence of copolymer precipitation.

SEM images revealed the morphologies of the obtained precipitate powder over a range of 33-75 mol \% APS. Some irregular particles with a wide size distribution are observed in Figure 1a when 33 mol \%
APS used. This suggests that there are two populations of particles, which a population of agglomerates accompany individual particles. The size of individual particles is much smaller than that of the agglomerates. Large particles may be caused by hydrophobic agglomeration at high phenyl fraction and high copolymer concentration, or by hard agglomeration during drying. The little-agglomerated particles with an average size of approximately $150 \mathrm{~nm}$ are observed in Figure 1b when APS mole percentage up to about $50 \mathrm{~mol} \%$. Exact amino group content at particle surface may provide additional stabilization through steric and charge repulsion to suppress the formation of aggomerates. ${ }^{13}$ With increase of APS up to $75 \mathrm{~mol} \%$, large spheroidal particles and much agglomeration induced by strong interaction of hydrogen bond due to high amino molar fraction in the copolymer occur (as in Figure 1c). It is interesting that particle size initially decreases and then increases with the APS ratio. Differences in the characteristics of final particles may be mainly attributed to variation in the hydrophobichydrophilicity of the copolymer particles, predominantly determined by the molar fraction of the incorporated aminopropyl units.

Various total monomer concentrations were performed at maintaining the equal molar ratio of PTES and APS. As observed from Figure 2a, rather uniform and little-aggregated smooth sphere particles of approximately $300 \mathrm{~nm}$ diameter occur at $8 \mathrm{wt} \%$ monomer concentration $(\mathrm{C}: 2.51 \mathrm{mmol} / \mathrm{g}$ by elemental analysis). Average particle size increases slightly and size distribution becomes broad up to 16 wt $\%$ in Figure $2 b$ (C: $2.75 \mathrm{mmol} / \mathrm{g}$ by elemental analysis). Nevertheless continuous particle growth is not observed, the occurring becomes smaller and less regular up to $20 \mathrm{wt} \%$ (Figure 1b). Amino group content increases slightly with total monomer concentration. In consideration of the results in Figure 1c (about $8 \mathrm{wt} \%$ PTES), spheroidal particles should be formed only at lower PTES comonomer concentration. The reason may be that the growth rate of particles is larger than the nucleation rate, and uniform growth leads to spheroidal topology. Increase in monomer supply causes increase in particle size, while semicontinuous monomer addition leads to continuous nucleation to give broad dispersity particles. Further increase in monomer concentration particularly APS concentration induces larger nucleation rate and the appearance of supersatuation level results in decrease in final particle size.

The effect of SDS concentration on the co-condensation of APS and PTES (1:1 molar ratio) over the range $0.25-4 \mathrm{wt} \%$ was investigated. Fine and coarse particles were seen in the reaction system at 0.25 wt $\%$ SDS concentration, indicating rapid agglomeration. SEM images further confirm the presence of 


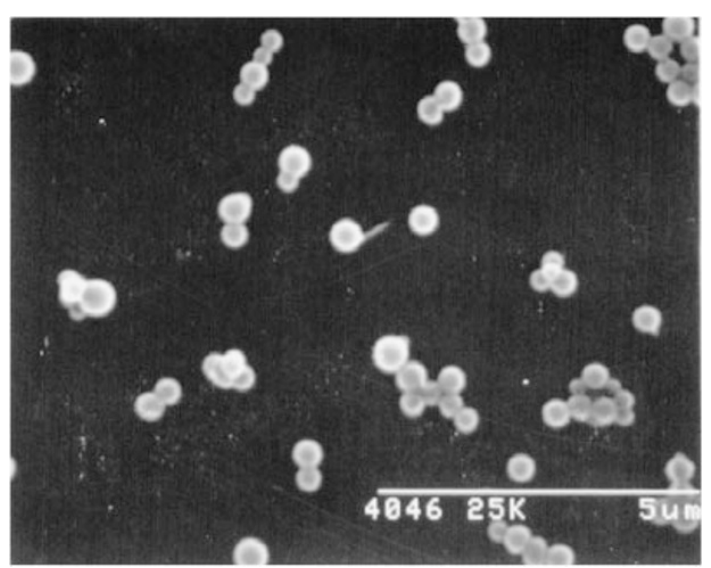

(a)

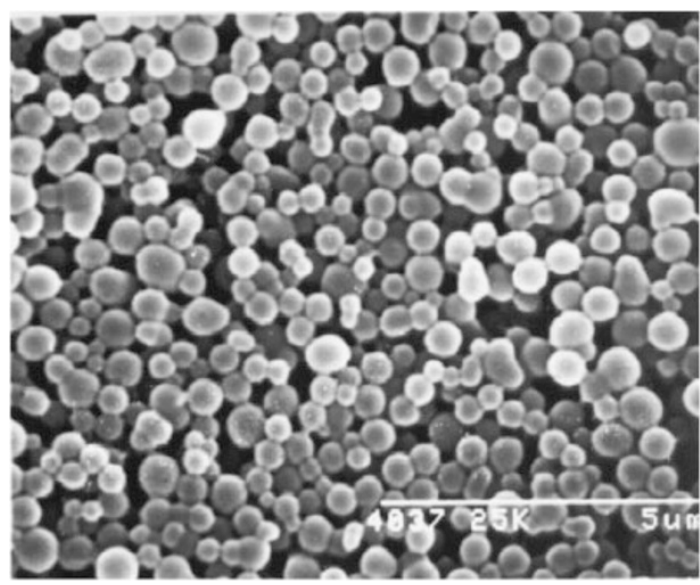

(b)

Figure 2. SEM images of samples at various total monomer concentrations (APS:PTES $=1: 1$, molar ratio) (a) $8 \mathrm{wt} \%$ and (b) $16 \mathrm{wt} \%$.

serious agglomeration (Figure 3a), which may originate from lack of SDS surfactant available to stabilize nucleated copolymer particles, and primary particles aggregate into larger stable particles. On increasing SDS to $1.0 \mathrm{wt} \%$, little agglomeration is observed and the particles disperse uniformly (Figure 1b). At $4 \mathrm{wt} \%$ (Figure 3b) particle size and agglomeration further decrease. This indicates that a large amount of SDS in the aqueous phase favors the stability of nucleation throughout the co-condensation. The larger the SDS concentration, the lower the agglomeration.

In addition to suitable control of APS/PTES mole ratios, total monomer concentration and SDS concentration, experimental conditions such as temperature, monomer addition mode and catalyst ATMOH concentration affect the hydrolytic co-condensation.

The structures of the products were determined from spectral FT-IR and ${ }^{29} \mathrm{Si} \mathrm{CP} / \mathrm{MAS}$ NMR data. Figure 4 shows FT-IR spectra of the samples of $\mathrm{SA}_{3}$ (Figure 4a) and $\mathrm{SA}_{5}$ (Figure 4b). They both exhibit well-defined phenyl absorption bands at: 3050, 3070

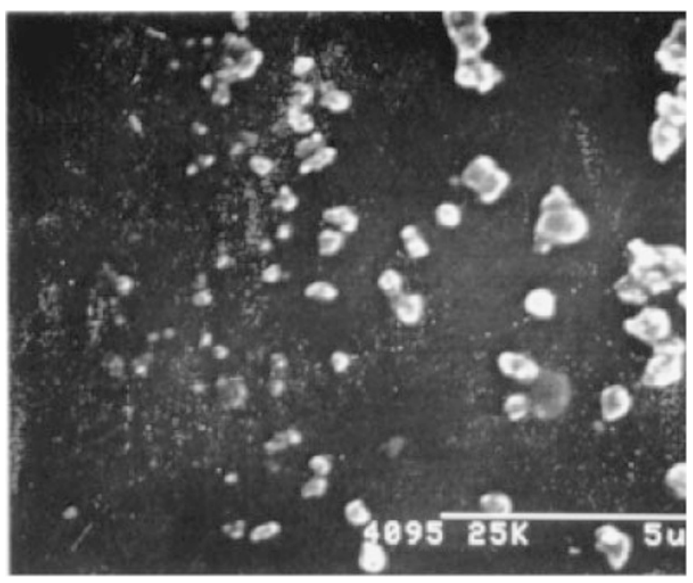

(a)

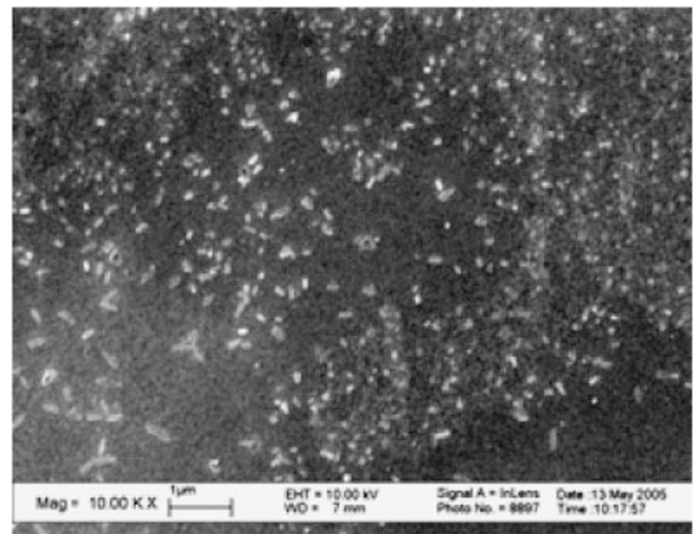

(b)

Figure 3. SEM images of samples at different SDS concentrations (a) $0.25 \mathrm{wt} \%$ and (b) $4 \mathrm{wt} \%$.

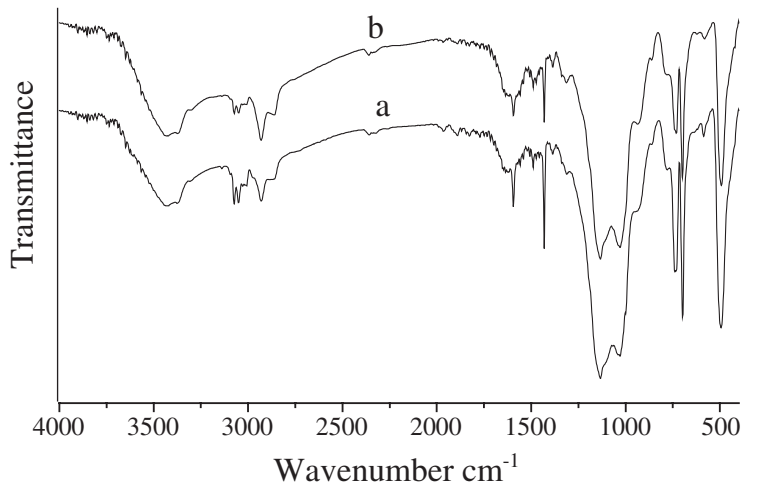

Figure 4. FT-IR spectra of samples (a) $\mathrm{SA}_{3}$ and (b) $\mathrm{SA}_{5}$.

$\left(v_{\mathrm{C}-\mathrm{H}}\right), \quad 1491, \quad 1595 \mathrm{~cm}^{-1} \quad\left(v_{\text {benzene ring }}\right), \quad 1430 \mathrm{~cm}^{-1}$ $\left(\delta_{\mathrm{C}-\mathrm{H}}\right), 697,740 \mathrm{~cm}^{-1}\left(\gamma_{\mathrm{C}-\mathrm{H}}\right)$, and $\mathrm{NH}_{2}\left(\mathrm{CH}_{2}\right)_{3}$ absorption bands at: $3550-3200 \mathrm{~cm}^{-1}\left(v_{\mathrm{N}-\mathrm{H}}\right.$, two peaks), $1640-1590 \mathrm{~cm}^{-1}\left(\delta_{\mathrm{N}-\mathrm{H}}\right.$, obscured to some degree by the strong absorptions characteristic of benzene ring bands), $2931\left(v_{\mathrm{C}-\mathrm{H}}\right)$. Although the absorption bands due to hydrogen bonding also appear at 3550-3200 $\mathrm{cm}^{-1}$, one double peak is characteristic of amino absorption band. The presence of $\mathrm{Si}-\mathrm{O}-\mathrm{Si}$ linkages 
is supported by intense absorptions at 1140-1000 $\mathrm{cm}^{-1}\left(v_{\mathrm{Si}-\mathrm{O}-\mathrm{Si}}\right)$. Compared with those of SA3, $\mathrm{NH}_{2}-$ $\left(\mathrm{CH}_{2}\right)_{3}$ absorption bands of SA5 become stronger and phenyl absorption bands correspondingly get weaker. Thus, the sample molecules are phenyl and aminopropyl silsesquioxane coploymer, and more aminopropyl groups are incorporated in SA5.

${ }^{29} \mathrm{Si} \mathrm{CP} / \mathrm{MAS}$ NMR spectra provide significant information about hybrid silsesquioxane frameworks. According to the spectrum of the hydrolytic condensation of PTES in a Stöber-like method, ${ }^{9}$ the peaks of pheny $\mathrm{T}^{3}$ and $\mathrm{T}^{2}$ units were at -79.1 and $-68.0 \mathrm{ppm}$. The spectrum from the hydrolytic condensation of APS alone by evaporating water at about $70^{\circ} \mathrm{C}$ and drying under vacuum exhibits two peaks at -71.0 and $-62.6 \mathrm{ppm}$, assigned to aminopropyl $\mathrm{T}^{3}$ and $\mathrm{T}^{2}$ units (Figure 5a). The peaks corresponding to the formation of $\mathrm{T}^{1}$ and $\mathrm{T}^{0}$ species are insignificant in the spectra. Therefore $\mathrm{T}^{3}$ species are in majority and $\mathrm{T}^{2}$ species are in minority for the hydrolytic condensation of PTES and APS under TAMOH catalyst $(\mathrm{pH}=$ 12.6). Two large peaks appear in the spectra of $\mathrm{SA}_{3}$ and $\mathrm{SA}_{5}$ (Figures $5 \mathrm{~b}$ and $5 \mathrm{c}$ ). It is out of question that one peak at $-84.1 \mathrm{ppm}$ is assigned to phenyl $\mathrm{T}^{3}$ units, where chemical shift variation of $c a$. $-5 \mathrm{ppm}$ may be induced by the change in the $\mathrm{Si}-\mathrm{O}-\mathrm{Si}$ bond angle due to different species $\mathrm{T}-\mathrm{T}$ bonding. The other at -72.6 ppm may be assigned to the superimposition of aminopropyl $\mathrm{T}^{3}$ units in majority and phenyl $\mathrm{T}^{2}$ units in minority, where chemical shift variation is $-1.6 \mathrm{ppm}$. A shoulder peak at $c a$. $-62 \mathrm{ppm}$ in Figure $5 \mathrm{c}$ is obvious, indicating small amount of aminopropyl $\mathrm{T}^{2}$ units at higher APS mole percentages. The peak at -84.1 $\mathrm{ppm}$ is higher than at $-72.6 \mathrm{ppm}$ in the spectrum of $\mathrm{SA}_{3}$ whereas the former is lower than the latter in the spectrum of $\mathrm{SA}_{5}$. This shows the fraction of aminopropyl groups in $\mathrm{SA}_{5}$ is higher than that in $\mathrm{SA}_{3}$. This is consistent with FT-IR analysis.

TGA traces in $\mathrm{N}_{2}$ of $\mathrm{SA}_{3}$ and $\mathrm{SA}_{5}$ is shown in Figure 6 . The weight loss prior to $180^{\circ} \mathrm{C}$ for $\mathrm{SA} 3$ is $3.3 \%$ (Figure $6 \mathrm{a}$ ), this being mainly associated with condensation reaction of residual silanols and ethoxy groups. A weight loss of $1.7 \%$ is observed at 180 $280^{\circ} \mathrm{C}$ due to decomposition of residual SDS, followed by a large weight loss of $5.8 \%$ from 280 to $450{ }^{\circ} \mathrm{C}$ assigned to decomposition of aminopropyl groups. The thermal degradation of siloxane network is maximum at $450-700{ }^{\circ} \mathrm{C}$, amounting to $23.6 \%$ equivalent to organic moieties-phenyl groups in the sample. $\mathrm{SA}_{5}$ (Figure 6b) exhibits a weight loss of $9.5 \%$ at temperature below $280^{\circ} \mathrm{C}$ attributable to residual silanols and ethoxy groups and SDS. A rapid weight loss of $13.6 \%$ occurs from 280 to $450^{\circ} \mathrm{C}$, an increase by $7.8 \%$ than $\mathrm{SA}_{3}$, and a weight loss of $19.3 \%$ over the range of $450-700^{\circ} \mathrm{C}$ is found, a reduc-

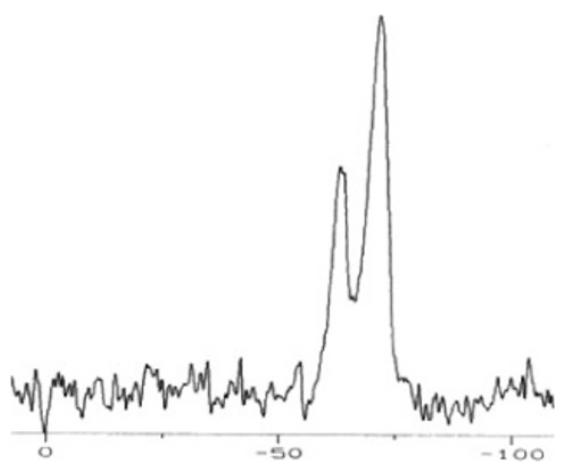

(a)

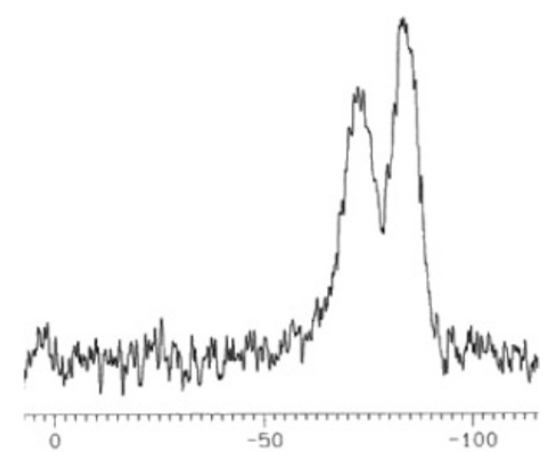

(b)

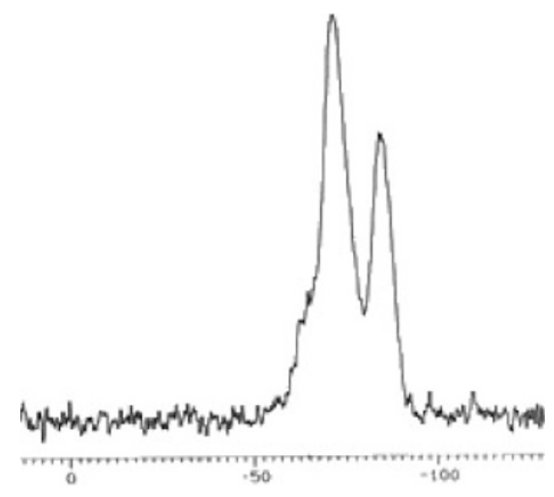

(c)

Figure 5. ${ }^{29} \mathrm{Si} \mathrm{CP} / \mathrm{MAS} \mathrm{NMR}$ spectra of samples (a) $\mathrm{SA}_{6}$; (b) $\mathrm{SA}_{3}$; (c) $\mathrm{SA}_{5}$.

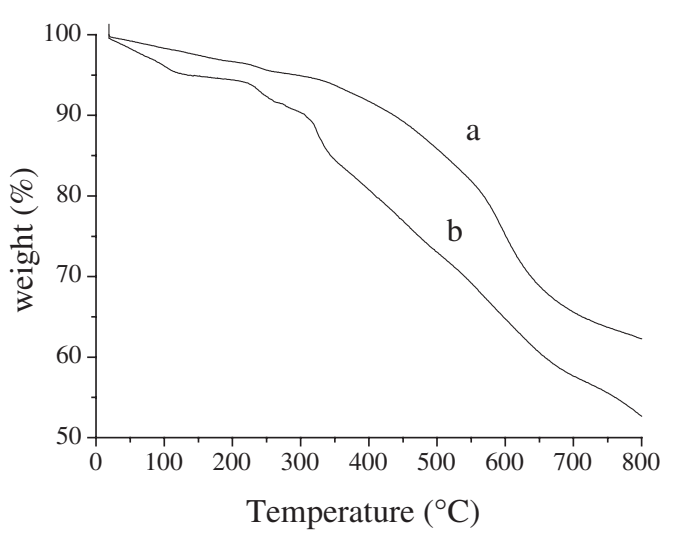

Figure 6. TGA traces of samples (a) $\mathrm{SA}_{3}$ and (b) $\mathrm{SA}_{5}$. 
tion by $4.3 \%$ than $\mathrm{SA}_{3}$. Total weight loss is $42.4 \%$ and more aminopropyl groups induce decrease in thermal stability of the copolymer. The TGA curves also give insight into that the copolymer in $\mathrm{SA}_{5}$ contains more aminopropyl and less phenyl groups than in $\mathrm{SA}_{3}$.

\section{CONCLUSIONS}

Poly(aminopropyl/phenyl)silsesquioxane was prepared by the hydrolytic co-condensation of PTES and APS under TAMOH catalysis in the presence of SDS. The copolymer takes on high amino group content. Copolymer particles were influenced to large degree by PTES/APS monomer ratios, total monomer and SDS concentration. Spheroidal particles were formed at lower PTES concentration. The incorporation of appropriate amounts of aminopropyl groups into the copolymer suppressed the formation of agglomerates and reduced the final particle size by an enhancement in particle stabilization. The presence of SDS favored the stability of nucleation throughout the co-condensation process. SEM images revealed manifold morphologies of the copolymer precipitate powder. ${ }^{29} \mathrm{Si} \mathrm{CP} / \mathrm{MAS}$ NMR spectroscopy and FTIR analysis confirmed the occurrence of co-condensation between APS and PTES. The TGA curves indicated that more aminopropyl groups induce decrease in thermal stability of the copolymer.

Acknowledgment. This research work was supported by a grant from the Key Project in Science and Technology of Guangzhou (No. 2004Z3-D0061) and from the Foundation for High Level University Construction of South China Universities of Technology (No. B09-333).

\section{REFERENCES}

1. R. H. Baney, M. Itoh, A. Sakakibara, and T. Suzuki, Chem. Rev., 95, 1409 (1995).

2. K. M. Kim, T. Ogoshi, and Y. Chujo, J. Polym. Sci., Part A: Polym. Chem., 43, 473 (2005).

3. M. C. Gravel, C. Zhang, M. Dinderman, and R. M. Laine, Appl. Organomet. Chem., 13, 329 (1999).

4. Y. Kaneko, N. Iyi, T. Matsumoto, K. Fujii, K. Kurashima, and T. Fujita, J. Mater. Chem., 13, 2058 (2003).

5. F. J. Feher and K. D. Wyndham, Chem. Commun., 3, 323 (1998).

6. D. R. Bujalski, H. P. Chen, G. A. Zank, and K. Suo, Macromolecules, 36, 3529 (2003).

7. A. Matsuda, T. Tanaka, K. Tadanaga, T. Minami, and M. Tatsumisago, J. Mater. Sci., 39, 903 (2004).

8. a) S. E. Tunney, G. A. Rakes, and B. K. Coltrain, U.S. Patent 5384376 (1995).

b) S. E. Tunney, G. A. Rakes, and B. K. Coltrain, Chem. Abstr. 123, P145607w (1995).

9. S. M. Liu, X. M. Lang, H. Ye, S. J. Zhang, and J. Q. Zhao, Eur. Polym. J., 41, 996 (2005).

10. C. Ma and Y. Kimura, Polym. J., 34, 709 (2002).

11. C. Ma, I. Taniguchi, M. Miyamoto, and Y. Kimura, Polym. J., 35, 270 (2003).

12. N. Tsubokawa, T. Iida, and T. Takayama, J. Appl. Polym. Sci., 75, 515 (2000).

13. R. M. Ottenbrite, J. S. Wall, and J. A. Siddiqui, J. Am. Ceram. Soc., 83, 3214 (2000). 